\title{
Characteristic Exponents and Strange Attractors
}

\author{
Sidnie Dresher Feit* \\ Department of Mathematics, Yale University, New Haven, Connecticut 06520, USA
}

\begin{abstract}
Iterates of maps in the family $f(x, y)=\left(y+1-A x^{2}, B x\right)$ (see [1]) are investigated. Characteristic exponents $C_{p}=\lim _{n \rightarrow \infty}(1 / n) \log \left\|d f^{n}(p)\right\|$ are estimated numerically. Further numerical investigations indicate that finite $C_{p}>0$ corresponds to a strange attractor. When $C$ values are calculated for $B$ fixed and $A$ in an interval, one finds dispersed among $C>0$ values many small subintervals for which $0>C$. On each such subinterval there appear to be attractors of period $k, 2 k, 4 k, \ldots$ the period doubling as $A$ increases. Many different values of $k$ have been observed. A theorem is proved for $A>0,1>B>0$ describing an explicit compact set $K$ (depending on $A$ and $B$ ) such that all non-divergent asymptotic behavior takes place in $K$.
\end{abstract}

\section{Introduction}

Let $f$ be a mapping of $R^{m}$ to $R^{m}$ defining a "time evolution" $p_{n}=f\left(p_{n-1}\right)$. By sensitive dependence on initial condition we mean that for $q_{0}$ near $p_{0}$ the distance $\left\|p_{n}-q_{n}\right\|$ is growing rapidly as $n$ increases (cf. Ruelle $[4,5]$ ).

If $f$ is differentiable, we can define this concept as follows: denote $f^{n}=f \circ \ldots \circ f$, i.e. the $n$-th iterate of $f$ and let $d f^{n}(p)$ be the derivative of $f^{n}$ at $p$, i.e. the $m \times m$ matrix $\left(\partial f_{i}^{n} / \partial x_{j}\right)$. Then $f$ has sensitive dependence on initial condition for a set $U$ if for each $p \in U$

1) the set $\left\{f^{n}(p) n=0,1, \ldots\right\}$ is bounded

2) $\lim _{n \rightarrow \infty}(1 / n) \log \left\|d f^{n}(p)\right\|=C_{p}>0$.

In this paper we present the results of numerical experiments performed on a particular family of diffeomorphisms mapping $R^{2}$ to $R^{2}$. This family was discussed

* This work was supported by the Institut des Hautes Etudes Scientifiques in France and by Yale University. I wish to express my gratitude to Prof. D. Ruelle for suggesting this investigation, and for his helpful comments, to Prof. S. Newhouse for many informative conversations, and to the Yale Department of Computer Science for the use of its extraordinary GEM computer system 
(A)

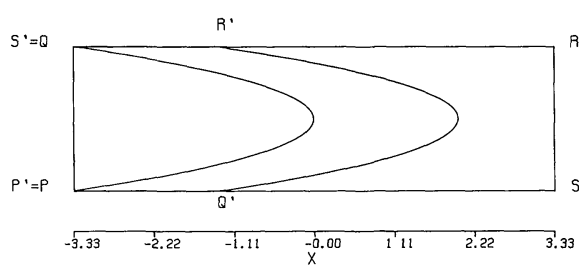

(C)

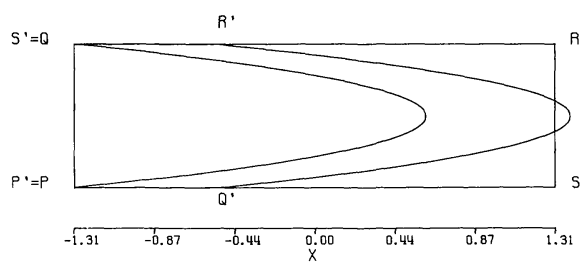

(B)

$A=0.98$

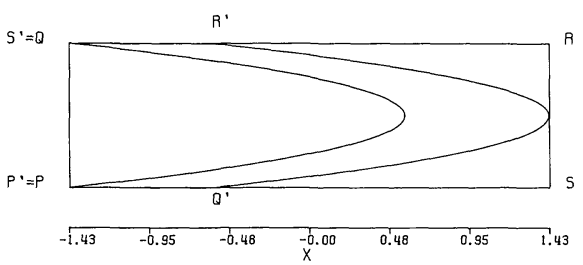

(D)

$A=1.35$

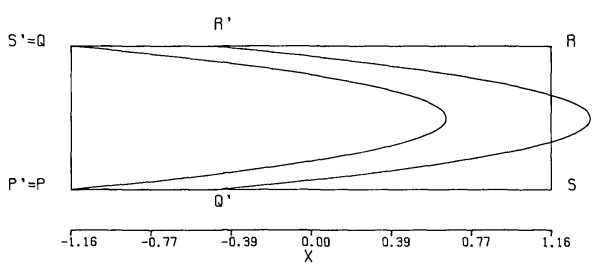

Fig. 1. Rectangles and their images $B=0.3$

by Henon in $[1]^{1}$. The numerical experiments which will be discussed in the present work appear to indicate that characteristic exponents exist for the Henon family of maps and for other polynomial maps as well, and that the value of $C_{p}$ is determined by an "attractor" asymptotically approached by iterates of $p$.

Throughout Sections 2-6 we shall only consider maps for which $A>0$ and $1>B>0$. We shall have a few remarks to make about other $A$ and $B$ values in Section 7.

\section{Some General Properties of the Maps $f(x, y)=\left(y+1-A x^{2}, B x\right)$}

In order to visualize the action of one of these maps, note that vertical lines are mapped to horizontal lines, while (for $A>0$ ) horizontal lines map to parabolas opening to the left. Each $f$ for which $A>0,1>B>0$ has two fixed points

$$
x=\frac{(B-1) \pm \sqrt{(1-B)^{2}+4 A}}{2 A}, \quad y=B x .
$$

One of these $x$ coordinates is negative and the other is positive. Denote the negative $x$ value by $-r$. In Figure 1 are displayed rectangles with vertices $( \pm r, \pm B r)$ and the images under $f$ of these rectangles when $B=0.3$ and $A=0.3$, $0.98,1.12$, and 1.35 respectively.

1 Henon pointed out that the maps $f(x, y)=\left(y+1-A x^{2}, B x\right)$ where $A$ and $B$ are parameters, model important properties of a Poincaré map for a three dimensional Lorenz flow studied numerically by Pomeau in 1976. Namely, there is stretching in one direction, an area is folded over itself, and the Jacobian of the mapping is constant (here equal to $-B$ everywhere). Furthermore, these maps are a canonical form for the most general quadratic maps of $R^{2}$ to $R^{2}$ having constant Jacobian. Henon devoted a great deal of attention to the map for which $B=0.3$ and $A=1.4$ 
(A) NON DIVERGENT POINTS FOR

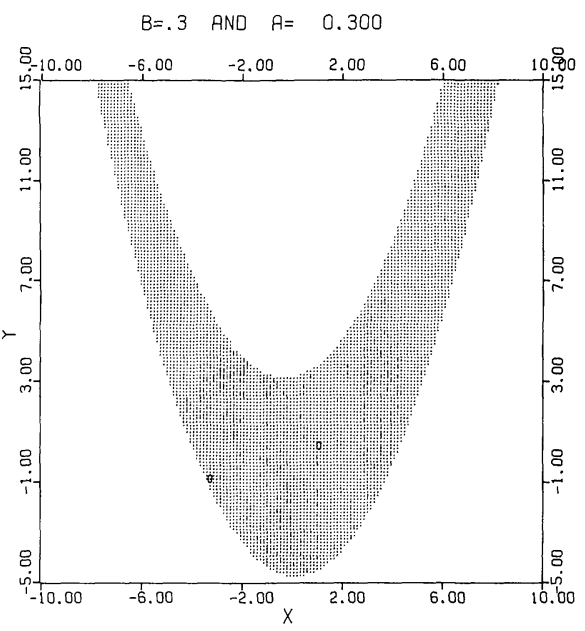

(C)

NON DIVERGENT POINTS FOR

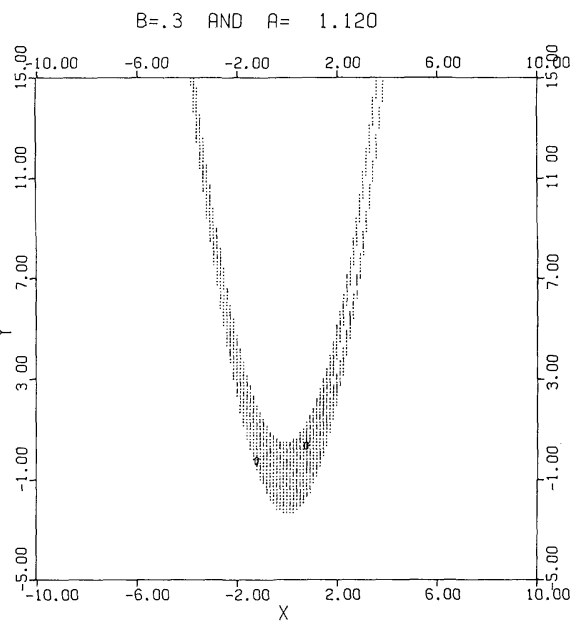

(B)

NON DIVERGENT POINTS FOR

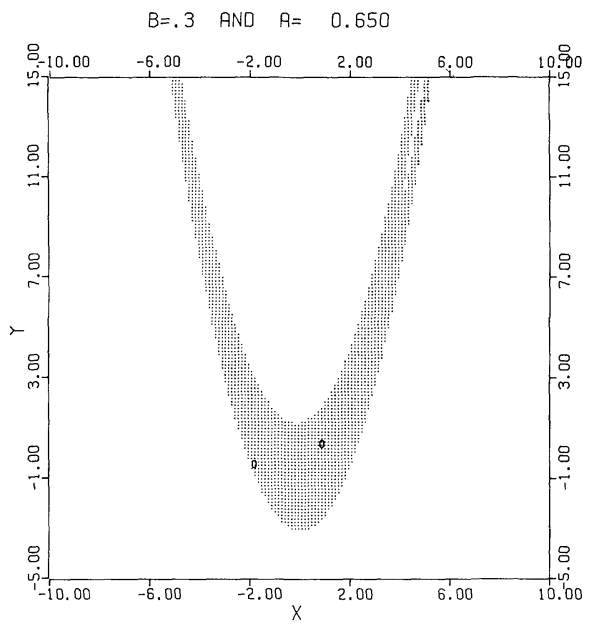

(D) NON DIVERGENT POINTS FOR

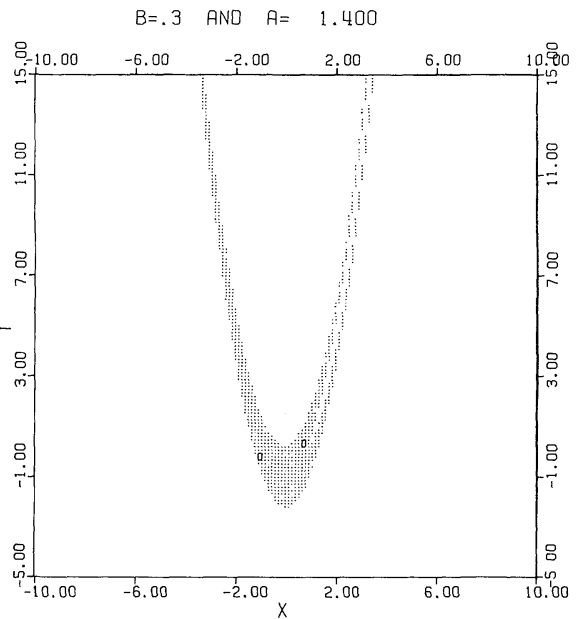

Fig. 2

The derivative of $f$ is $\left(\begin{array}{cc}-2 A x & 1 \\ B & 0\end{array}\right)$. If we calculate the absolute values of the eigenvalues of the derivative, the smaller is always less than 1 , but the larger is, respectively $<,=$, or $>1$ depending on whether $|x|$ is $<,=$, or $>(1-B) / 2 A$.

\section{Divergent and Non-Divergent Points}

Before discussing the numerical results, it might be of value to have an overall picture of the iteration of $f(x, y)=\left(y+1-A x^{2}, B x\right)$. Proofs of the following observations appear in Appendix I. 


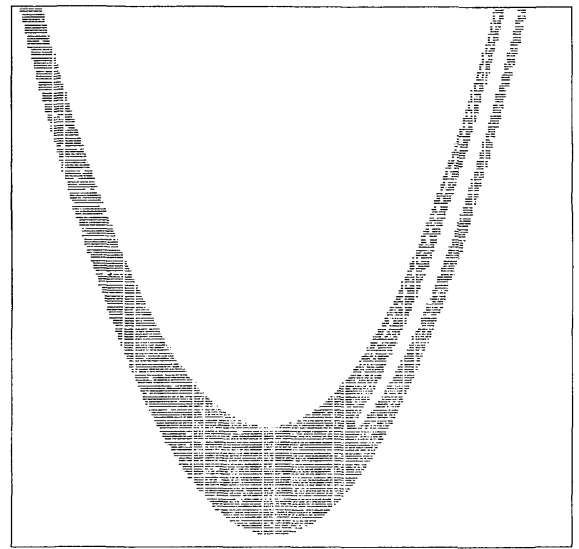

Fig. 3. Non divergent points for $A=1.4$ and $B=0.3$

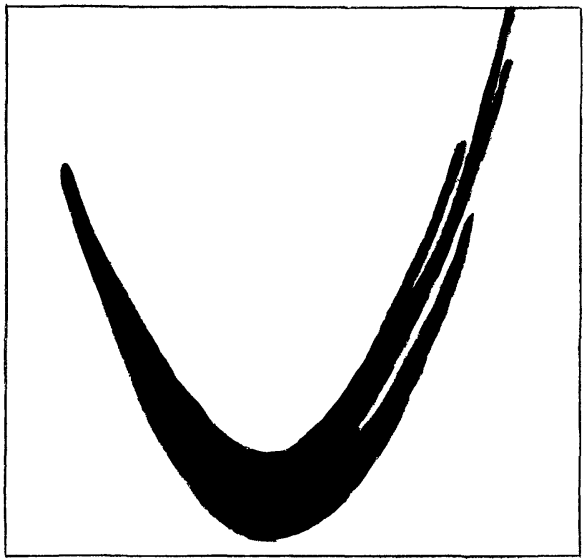

Fig. 4. Schematic picture of connected non divergent set

Throughout this section we assume that $A>0,1>B>0$.

Let us call a point $\left(x_{0}, y_{0}\right)$ divergent if $\lim _{n \rightarrow \infty} f^{n}\left(x_{0}, y_{0}\right)=(-\infty,-\infty)$. The set of divergent points can in fact be described explicitly. Let

$$
Q=\{(x, y) \mid x<-1-\sqrt{1+4 A}, y<0\} .
$$

It is easy to show that $f(Q) \subset Q$ and that, further, the set of all divergent points is the open connected set $D=\bigcup_{n=0}^{\infty} f^{-n}(Q)$. Moreover, one can construct a compact set $K$ bounded by a polygon (depending on $A$ and $B$ ) such that if $\left(x_{0}, y_{0}\right)$ is not divergent, then there is an $n_{0}>0$ such that $f^{n}\left(x_{0}, y_{0}\right) \in K$ for all $n>n_{0}$. In order to study the asymptotic behavior of a given $f$, it suffices to examine iterates of points in the intersection of $K$ with the complement of $D$. This intersection is a compact $f$-invariant set. 
(A)

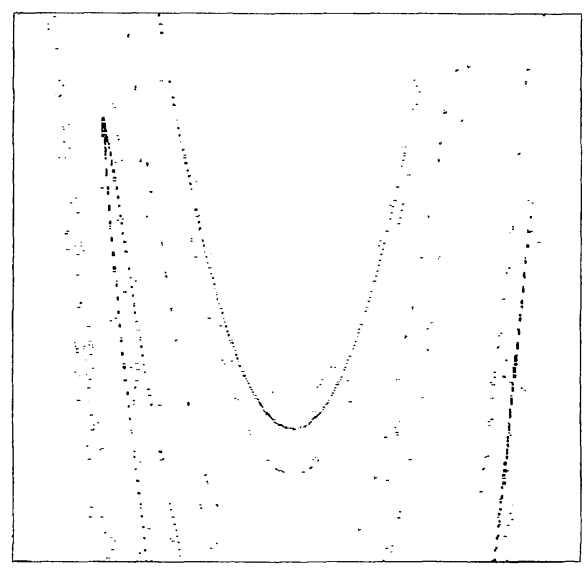

(C)

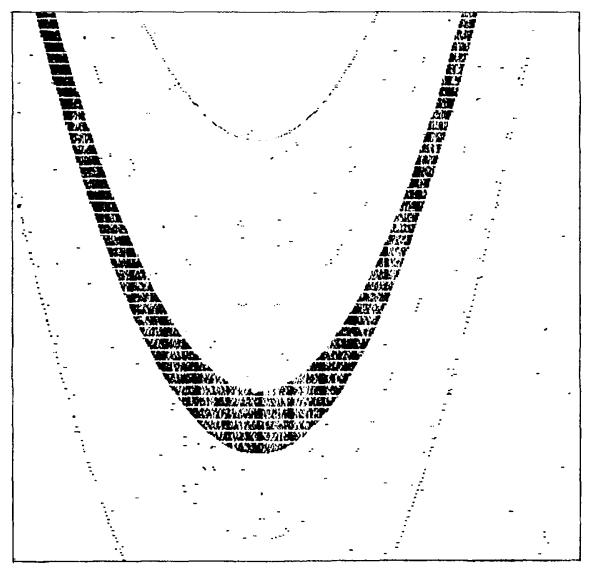

(B)

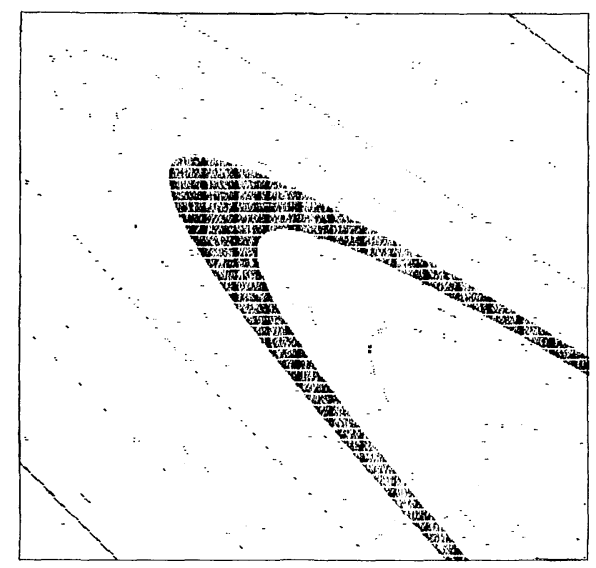

(D)

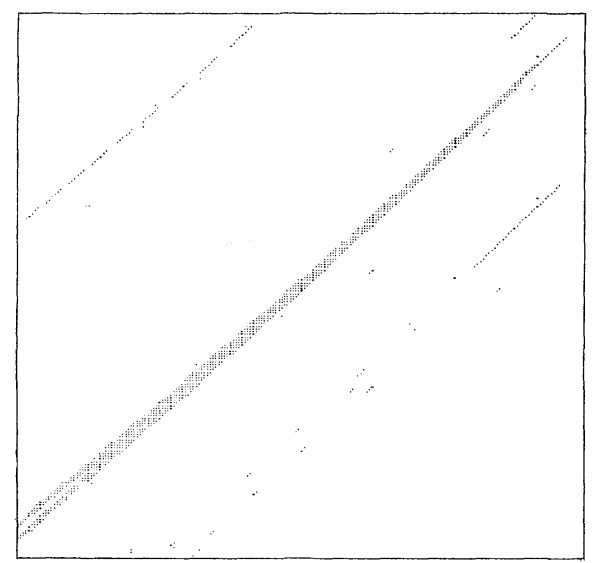

Fig. 5. A Non divergent points for $A=2.6577$ and $B=0.3$. B $A=2.6577$ and $B=0.3$; detail near left point in orbit. $\mathbf{C} A=2.6577$ and $B=0.3$; detail near center point in orbit. $\mathbf{D} A=2.6577$ and $B=0.3$; detail near right point in orbit

The shaded regions in Figures 2A-D are parts of the sets of non-divergent points for $B=0.3$ and $A=0.3,0.65,1.12$, and 1.4 . The fixed points are marked in the figure by circles. Figure 3 is a more detailed picture for $B=0.3$ and $A=1.4$. These pictures are somewhat misleading however, and in Figure 4 we have drawn a picture which is distorted with respect to scale, but which gives a truer idea of how the shape develops. (One can prove that the boundary of $D$ contains the stable manifold through the left fixed point. This winds around quite a bit.) In Figure 5A we display a portion of the rather wilder looking non-divergent set for $B=0.3$ and $A=2.6577$, where $f$ has a period 3 attractor.

Figures 5B-D show neighborhoods of the three points of the attracting orbit $(0.0104 \ldots, \quad-0.2039 \ldots) \rightarrow(0.7957) \ldots, \quad 0.0031 \ldots) \rightarrow(-0.6798 \ldots, 0.2387 \ldots)$. 
ATTRACTOR FOR $B=.3 A=1.400$

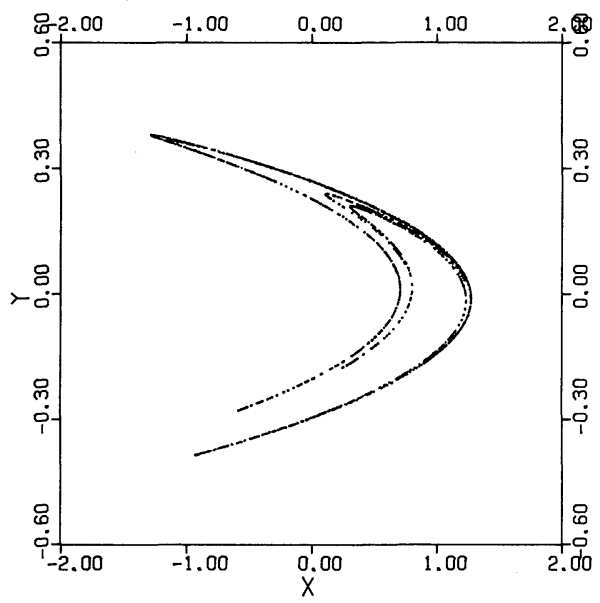

Fig. 6

Slightly larger values of $A$ for which there appear to exist strange attractors have similar looking non-divergent sets.

\section{The Characteristic Exponents}

Suppose that $f$ has an attracting orbit of period $k$. The eigenvalues of $d f^{k}$ are the same for any $p$ in the orbit, and an easy calculation shows that for almost any vector $v \neq 0,\left\|d f^{n}(p) v\right\|$ grows like $M e^{C n}$ where $M$ is some constant and $C=(\log |\lambda|) / k$ with $\lambda$ the eigenvalue of $d f^{k}$ of greatest absolute value. $\left(|\lambda|<1\right.$ since $f^{k}$ has an attracting fixed point.) Almost all points asymptotically approaching this attractor will haved the same characteristic exponent $C<0$.

In contrast, for $B=0.3$ and $A=1.4$, Henon numerically generated what appears to be a "strange" attractor (locally it appears that it could be a segment crossed with a Cantor set) by plotting thousands of iterates of $p=(0,0)^{2}$ (see Fig. 6). The characteristic exponent corresponding to this attractor is approximately 0.42 .

\section{Relationship between Characteristic Exponents and $\boldsymbol{A}$ Values}

The characteristic exponents of $f(x, y)=\left(y+1-A x^{2}, B x\right)$ are studied here by holding the parameter $B$ fixed, and tabulating values of $C$ versus several hundred values of $A$.

2 This example is especially interesting because Henon found a compact connected region $V$ containing the attractor, with $f(V) \subset V$. One may speculate as to whether there is a unique attractor for this map, which is equal to $\bigcap_{n} f^{n}(V)$. If this were so, the attractor would be a compact connected set containing the closure of the unstable manifold through the right fixed point (which lies in $V$ ). I performed an experiment in which thousands of distinct points in the region $K$ for this map were subjected to iteration, and each appeared to produce the same attractor, making the above conjecture somewhat convincing 


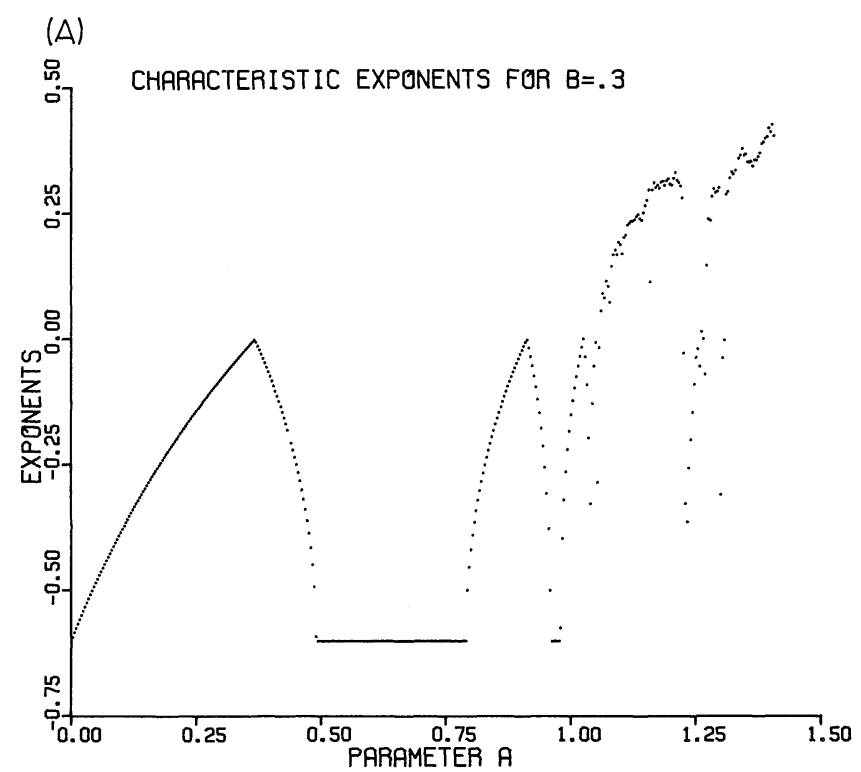

(B)

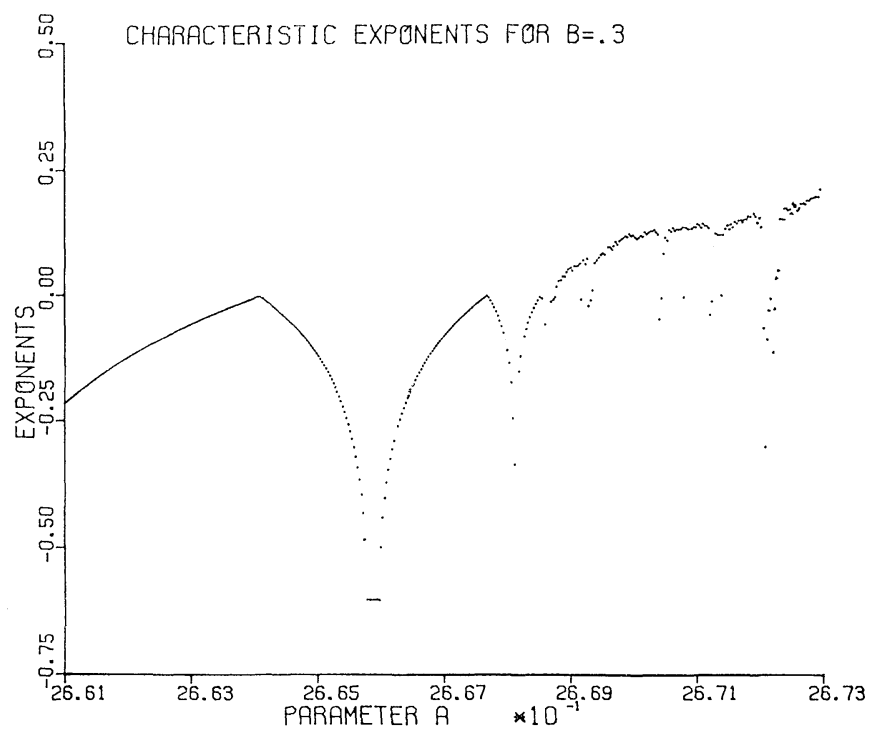

Fig. 7

For small $A$, of course, one does not need a computer to see what is going on; for $A=0$, all points in the plane converge to $(1 /(1-B), B /(1-B))$ which is the unique fixed point of $f$. When $A<(3 / 4)(1-B)^{2}$, the right fixed point of $f$ lies in the region $|x|<(1-B) / 2 A$ where both eigenvalues of the derivative have absolute value less than 1 and this point is attracting. When $A=(3 / 4)(1-B)^{2}$ the larger 
eigenvalue has absolute value 1 and a bifurcation takes place with a period two attracting orbit being created. A period two orbit continues to attract until $A=(1-B)^{2}+(1+B)^{2} / 4$, where a period four attracting orbit appears.

As $A$ increases, further bifurcations create attracting orbits of periods $8,16, \ldots$ over a succession of intervals which have been determined numerically. (The size of these intervals appears to decrease geometrically.)

Perhaps a word should be included here about the method of computing $C$ values. An initial point $\left(x_{0}, y_{0}\right)$ and initial vector are chosen, and are iterated by $f$ and $d f$ respectively several thousand times, so as to allow the point to "approach" its asymptotic behavior. Now we let $v$ be a unit vector having the direction of the current vector image, and calculate $C \sim(1 / n) \log \left\|d f^{n} v\right\|$ (using the natural logarithm) for large values of $n^{3}$. When the point has been pulled toward a periodic attractor, convergence to the corresponding $C<0$ has been rapid. When $C>0$ we have sensitive dependence on initial condition, and even with the greatest of care, round off errors are inherent in the calculations. Nonetheless, one seems to follow a kind of "average" orbit around an attractor and consistent values of $C$ with three digit agreement were calculated even after 500000 iterations, taken from a variety of initial values.

In Figure 7A we see a plot of $C$ versus $A$ for $B$ fixed at 0.3 and $0 \leqq A<1.42$. The first positive $C$ value occurs at $A=1.058048 \ldots$. To the right of this $A$, although many $C>0$ appear, one also finds a scattering of subintervals on which $C<0$, each such $C$ corresponding to a periodic attractor. [The fact that a period 7 attractor occurred for $A=1.3$ was noted earlier by Parisi (private communication).] To the right of an $A$ value having $C<0$ and an attracting orbit of period $k$, it appears numerically that on an open interval of increasing $A$ values there will be a series of bifurcations to attracting orbits of periods $2 k, 4 k, 8 k \ldots$.

Furthermore, for almost any $A$ interval chosen at random within $(1.058, \ldots, 4.2)$ a computer search resulted in a subinterval on which $C<0$ and there existed a periodic attractor, suggesting that such attractors may be densely scattered throughout the interval.

For $A=1.0752$ the extremely interesting phenomenon of two distinct attractors was observed, one of period 24 , and the other (with $C \sim 0.1$ ) apparently strange ${ }^{4}$.

For $A \in(1.42,2.6575)$ it has not been possible to compute any $C$ values or find any attractors. All starting choices appear to diverge, causing one to suppose that either no attractors exist or that the area of non divergent points is so attenuated that the inaccuracies inherent in computer calculations perturb orbits out of the region; possibly one has a zero dimensional non-wandering set here.

However a new interval of interesting values was found as follows. Noting the special significance of the appearance of the first period three orbit for mappings of intervals (see [2]), we used numerical methods to find the smallest value of $A$

\footnotetext{
3 A single value of $C$ was generally calculated by iterating $f$ and $d f 3000$ times and then using an additional 3000 iterates for the actual averaging. As a check, many longer iterations, up to 500000, were used to determine whether $C$ was indeed a stable value for a particular attractor. Both the GEM system and the Yale IBM 370 were used in these calculations, all of which were performed in double precision $4 \quad$ According to the result of Newhouse [3], one should not be surprised at finding infinitely many periodic attractors for some of these maps
} 


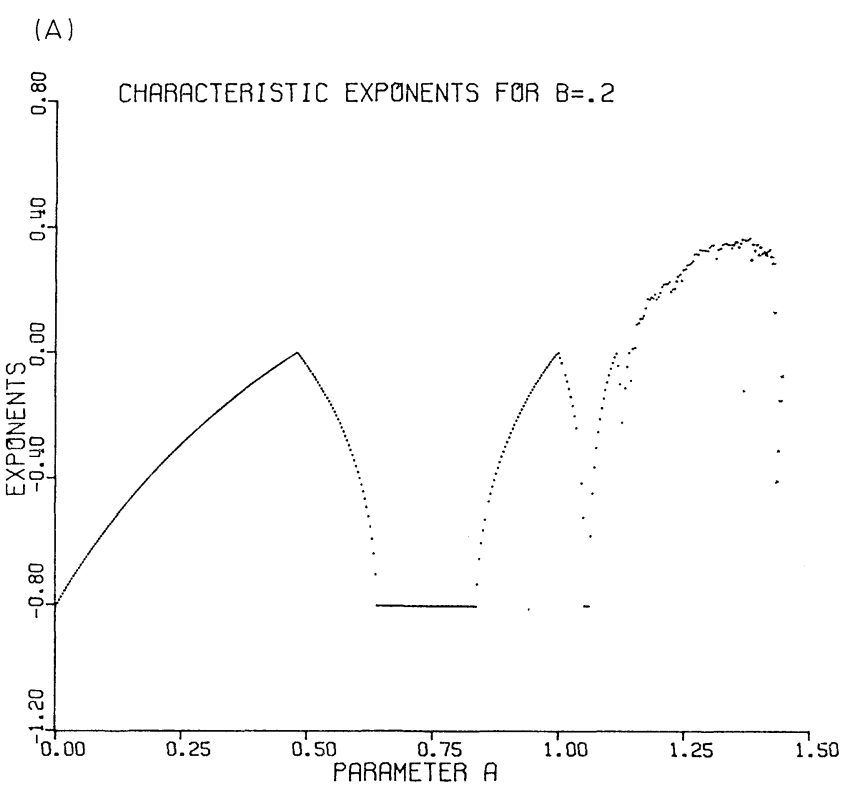

(B)

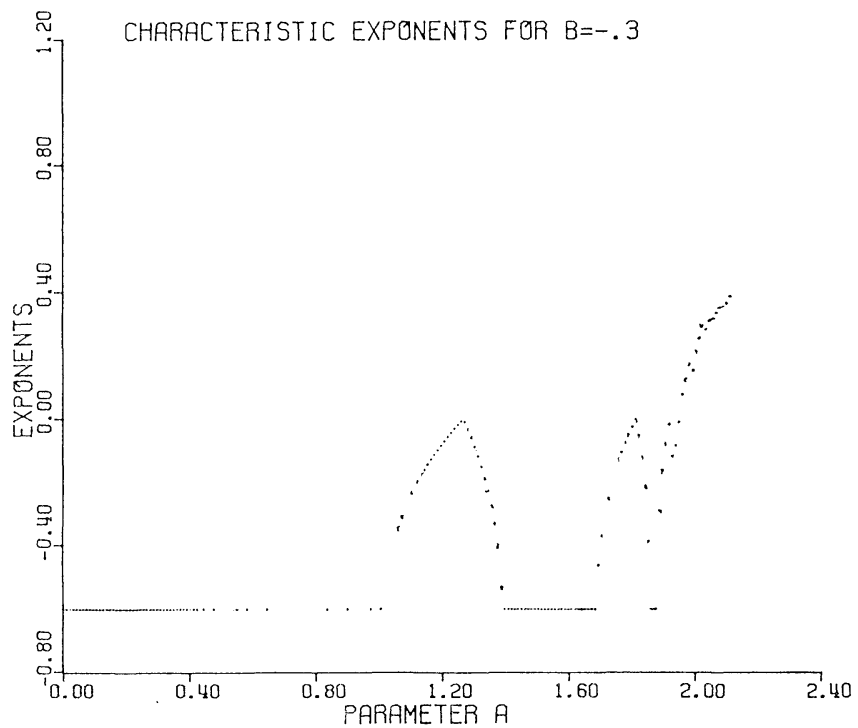

Fig. 8

$(B=0.3)$ for which a period three orbit occurs, namely $A=2.6575 \ldots$. On $(2.6575, \ldots, 2.67336 \ldots)$ we first find attracting orbits of period $3,6,12, \ldots$ and then at about $A=2.668$ we begin to find values of $C>0$, although again negative $C$ values corresponding to more periodic attractors are dispersed through the interval (see Fig. 7B). To the right of this interval it has not been possible to calculate any $C$ values or find any attractors. 


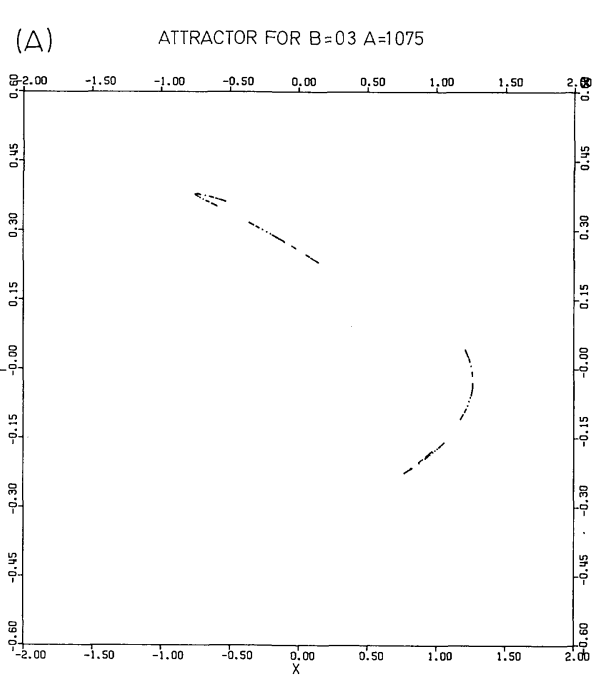

(C) ATTRACTOR FOR $B=.3 A=1.150$

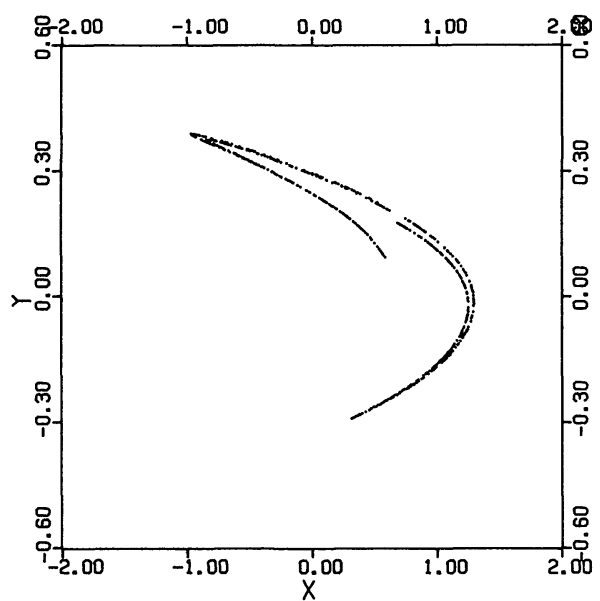

(B) ATTRACTOR FOR B=.3 $A=1.120$

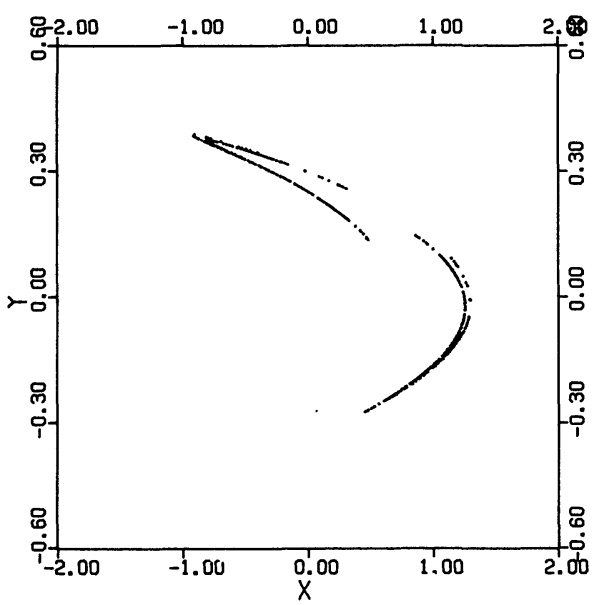

(D) ATTRACTOR FUR $B=.3 A=1.310$

Fig. 9

In Figure 8A $C$ is plotted versus $A$ for $A$ in the interval $(0,1.45)$ and for $B=0.2$. The behavior is similar to that observed in Figure 7A. (There is a period 3 attractor at $A=2.322222213$ and a second interval of $C$ values can be calculated for $A$ in an interval to the right of this point.)

\section{Strange Attractors}

Given values of $A, B$, and $p$ yielding $C>0$, one can plot the iterates of $p$ in order to observe the asymptotic behavior. In the figures generated for this paper the first 
3000 iterates are not plotted in order to avoid extraneous, non-asymptotic values. For each case for which the experimental plot was generated, a figure that could be a "strange" attractor developed; detailed sections concentrating on a small region near the attractor showed a characteristic pattern of many layers of line segments (cf. Henon [1] for $A=1.4$ and $B=0.3$ ). Also to be noted is the way in which these attractors straddle the regions in which the larger absolute value of eigenvalue for $d f$ takes on values $<,=$ and $>1$. [Recall that the value is 1 when $x=(1-B) / 2 A$.] Figures 9A-D display attractors for $B=0.3$ and $A=1.075,1.12,1.15$, and 1.31 .

\section{Some Further Calculations}

Negative values of $B$ also yield interesting non-divergent sets, attractors, and characteristic exponents. In Figure 8B, values of $C$ have been plotted for $A \in(0,2.1)$ and $B=-0.3$ using the initial value $\left(x_{0}, y_{0}\right)=(0.72,-0.014)$. Again, strange attractors appear to correspond to $C>0$.

The family of functions $g(x, y)=\left(y+1-A x^{3}+D x, B x\right)$ also produced similar results for fixed positive $B$ and $D$. Stable values of $C$ were calculated for $B=0.3$, $D=1.0$, and $A \in(0,0.61)$. One can obtain a graph quite similar to Figure $7 \mathrm{~A}$ in appearance.

\section{Appendix I}

In the following, assume that $f(x, y)=\left(y+1-A x^{2}, B x\right)$ and $A>0,1>B>0$. Let $s$ be the negative solution of $A x^{2}+x-1=0$ i.e. $s=(-1-\sqrt{1+4 A}) / 2 A$. Also, denote $\left(x_{1}, y_{1}\right)=f\left(x_{0}, y_{0}\right),\left(x_{n}, y_{n}\right)=f^{n}\left(x_{0}, y_{0}\right)$.

Lemma 1. Let $Q=\{(x, y) \mid x<s, y<0\}$. Then $f(Q) \subset Q$. Further, if $\left(x_{0}, y_{0}\right) \in Q$ then $\lim _{n \rightarrow \infty} f^{n}\left(x_{0}, y_{0}\right)=(-\infty,-\infty)$.

Proof. We have $y_{1}=B x_{0}<0$. If $x_{0}=s-c$ then $x_{1}=y_{0}+1-A x_{0}^{2}<1-A x_{0}^{2}=1$ $-A s^{2}-(1+\sqrt{1+4 A}) c-A c^{2}<s-2 c-A c^{2}$.

Remark. If $x \leqq s, y<0$ or $x<s, y \leqq 0$ then $f^{n}(x, y) \rightarrow(-\infty,-\infty)$.

Lemma 2. Let $M_{u}=\{(x, y) \mid x<u, y<B u+B\}$. If $u \geqq(1+2 B) /(1-B)$, then $f\left(M_{u}\right) \subset M_{u-B}$.

Proof. Let $\left(x_{0}, y_{0}\right) \in M_{u}$. Then $x_{1}=y_{0}+1-A x_{0}^{2}<y_{0}+1<B u+B+1 \leqq u-B$. $y_{1}=B x_{0}<B u<B u+B-B^{2}$.

Corollary. Let $m=(1+2 B) /(1-B)$. Given $(x, y)$ there exists $n_{0}$ such that $f^{n}(x, y) \in M_{m}$ for all $n \geqq n_{0}$.

We shall now show that there are regions $R_{1}$ and $R_{2}$ such that $f\left(R_{1}\right) \subset Q$, $f^{2}\left(R_{2}\right) \subset Q$ and $K=\bar{M}_{m}-\left(Q \cup R_{1} \cup R_{2}\right)$ is compact. Thus all points either eventually map to $Q$ and stay there, or else stay in $K$. Once a point leaves $K$, it will map to $Q$ and never return. 


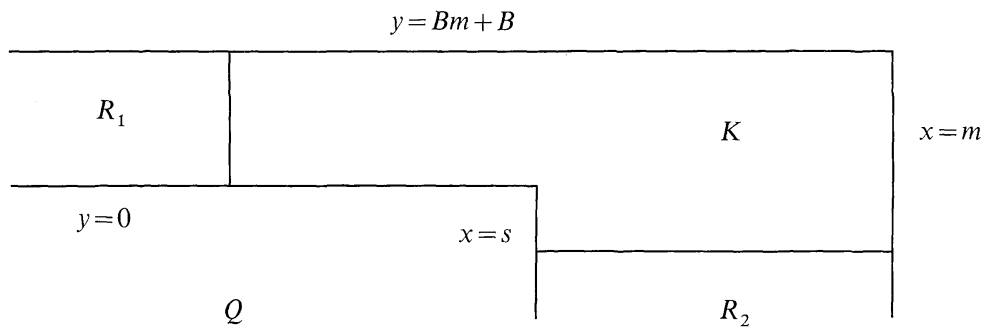

Lemma 3. (Region $\left.R_{1}\right)$. If $y_{0} \leqq B m+B$ and $x_{0}<-\sqrt{(B m+B+1-s) / A}$ then $f\left(x_{0}, y_{0}\right) \in Q$.

Proof. Since $x_{0}<0, y_{1}<0$ and $x_{1}<B m+B+1-(B m+B+1-s)=s$.

Lemma 4. (Region $R_{2}$ ). If $x_{0} \leqq m$ and $y_{0}<-1-\sqrt{(1+B m-s) / A}$ then $f^{2}\left(x_{0}, y_{0}\right) \in Q$.

Proof. $x_{1}<-\sqrt{(1+B m-s) / A}, y_{1} \leqq B m$, hence $y_{2}<0$ and $x_{2}=y_{1}+1-A x_{1}^{2}<B m$ $+1-(1+B m-s)=s$.

\section{References}

1. Henon, M. : A Two-dimensional mapping with a strange attractor. Commun. math. Phys. 50, 69-77 (1976)

2. Li, T., Yorke,J.: Period three implies chaos. Am. Math. Monthly 82, 985-992 (1975)

3. Newhouse, S. : Diffeomorphisms with infinitely many sinks. Topology 12, 9-18 (1974)

4. Ruelle,D.: Dynamical systems with turbulent behavior. (To appear in the Proceedings of the International Mathematical Physics Conference of Rome, 1977)

5. Ruelle,D.: Sensitive dependence on initial conditions and turbulent behavior of dynamical systems. (Talk presented at the Conference on Bifurcation Theory and Its Applications in Scientific Disciplines, 1977)

Communicated by J. L. Lebowitz

Received January 3, 1978 